\title{
ESTRUTURA E PROCESSO ASSISTENCIAL DE ENFERMAGEM AO PACIENTE COM CÂNCER ${ }^{1}$
}

NURSING ASSISTANCE PROCESS AND STRUCTURE FOR CANCER PATIENTS

\author{
Nilce Piva Adami ${ }^{2}$ \\ Maria Gaby Rivero de Gutiérrez ${ }^{3}$ \\ Amélia Maria Scarpa de A. Maranhão ${ }^{3}$ \\ Elizabeth Pinto Magalhães de Almeida ${ }^{4}$
}

\begin{abstract}
RESUMO: Esta pesquisa objetivou avaliar a estrutura para o atendimento ao paciente com câncer e o processo asssistencial em quimioterapia antineoplásica. Foi realizada em seis unidades de internação e uma ambulatorial de um hospital universitário, utilizando-se a observação sistematizada e entrevista semi-estruturada, no período de janeiro a junho de 1996. Constatou-se que existem deficiências na área física e instalações das unidades estudadas. O pessoal de enfermagem, de modo geral, não possui preparo formal em Oncologia, com exceção do Setor de Quimioterapia. Verificou-se falta de sistematização da assistência de enfermagem e diferenças entre as unidades estudadas no que tange aos aspectos técnicoorganizacionais da administração de antineoplásicos, bem como na interação entre profissionais e pacientes atendidos. As inadequações observadas poderiam ser minimizadas com uma política que contemple o aumento do financiamento do setor público da saúde, a implantação da sistematização da assistência e a inclusão da Oncologia na educação permanente do pessoal de enfermagem.
\end{abstract}

UNITERMOS: Enfermagem oncológica - Processo assistencial - Avaliação da estrutura.

\begin{abstract}
This study aimed at evaluating the structure for cancer patient assistance and antineoplasic chemotherapy assistance process. It has been performed at six internation units and one out-patient unit at a university hospital. Systematized observation and semistructured interview were used from January to June, 1996. It was noticed the there were some deficiencies in the physical area and installations of the studied units. Generally, nursing team had no formal training in Oncology, except for Chemotherapy Sector. The absence of a systematized nursing assistance and differences among the studied units as for technical organizational aspects for antineoplasic administration as well as patients and staff interaction has been observed. Inadequations observed could be minimized with such a politics which would be able to increase public sector financing, planting of systematized care and inclusion of Oncology in the permanent nursing team education.
\end{abstract}

KEYWORDS: Structure evaluation - Oncology nursing - Assistance process.

\footnotetext{
${ }^{1}$ Trabalho apresentado no $48^{\circ} \mathrm{CBEn}$. - São Paulo - SP, outubro de 1996.

2 Enfermeira Doutora em Enfermagem. Professor Adjunto da UNIFESP/EPM

${ }^{3}$ Mestre em Enfermagem Obstétrica e Neonatal. Professor Substituto da UNIFESP/EPM

${ }^{4}$ Enfermeira do Hospital São Paulo. Especialização em Enfermagem em Oncologia
} 


\section{INTRODUÇÃO}

O movimento pela qualidade nos serviços de saúde é um fenômeno mundial, em decorrência da crescente conscientização de que, na sociedade contemporânea, a qualidade é um requisito indispensável para a sobrevivência dos serviços de saúde e, mais importante ainda, segundo alguns, uma responsabilidade ética e social ${ }^{15}$.

Nesta concepção, o processo avaliativo deve ser entendido como um dos instrumentos da gestão desses serviços, a ser desenvolvido de forma contínua, indispensável para mensurar os esforços da instituição voltados para o alcance da qualidade, excelência, utilidade e relevância social.

Assim, a avaliação constitui-se num processo intencional, técnico e político, portanto, isento de neutralidade, auxiliado por diversas áreas do conhecimento e que pode ser aplicado a qualquer prática profissional, a uma organização, a uma rede de serviços, a um programa ou a uma atividade de saúde.

Face à importância atribuída, atualmente, à avaliação da qualidade nas instituições de saúde, este trabalho é parte integrante de um Projeto de Investigação sobre o Ensino e a Assistência de Enfermagem em Oncologia, financiado pelo CNPq.

\section{PROBLEMA, JUSTIFICATIVA E OBJETIVOS DO ESTUDO}

Tendo por base a vivência profissional das autoras, as questões que nortearam esta pesquisa foram: - A estrutura disponivel num determinado hospital universitário é adequada para o atendimento ao paciente com câncer? 0 processo assistencial de enfermagem em quimioterapia antineoplásica atende aos padrões determinados para o exercício dessa prática?

Estas indagações surgiram em decorrência da constatação de duas situações: a crise em que se encontra o Sistema Único de Saúde no Brasil, face ao financiamento insuficiente destinado ao setor, que afeta, significativamente, as instituições públicas desta área; a carência de estudos avaliativos sobre a assistência de enfermagem prestada aos usuários da instituição selecionada, considerando-se que o câncer representa um dos principais motivos de hospitalização e ocupa uma posição expressiva entre as causas de morbimortalidade no Estado de São Paulo e no país ${ }^{8}$.

Os objetivos estabelecidos para este estudo foram os de avaliar:

- a área física e instalações para o atendimento ao paciente com câncer e os recursos materiais para aplicação de quimioterapia antineoplásica, disponíveis em seis unidades de internação e na Unidade de Quimioterapia Ambulatorial de Adultos; 
- os recursos humanos de enfermagem que atuam nestas unidades;

- as normas técnicas relacionadas ao serviço de enfermagem;

- o sistema de informação sobre câncer;

- o processo assistencial em quimioterapia, desenvolvido pelo pessoal de enfermagem nas unidades selecionadas.

\section{A AVALIAÇÃO DA QUALIDADE EM SAÚDE}

Os Estados Unidos da América representam o país que se destaca em estudos e pesquisas avaliativas desde o início deste século, principalmente quanto 'a elaboração de padrões e métodos destinados à avaliação da qualidade da assistência à saúde ${ }^{3,10,12,16,18}$.

Em nosso país, de acordo com Arcucio e col. ${ }^{1}$, não existe experiência acumulada sobre a avaliação da qualidade dos serviços públicos de saúde e a pequena experiência desenvolvida refere-se, principalmente, à avaliação quantitativa, que tem se mostrado insuficiente para avaliar o conjunto, bastante complexo, de atribuições inerentes aos serviços de saúde.

\section{Significado}

A definição de qualidade dos serviços de saúde varia na dependência do quadro teórico-prático no qual se origina. Habitualmente, reflete as metas e os valores do sistema de saúde e da sociedade na qual este sistema se insere ${ }^{1}$. Conseqüentemente, essa definição deve ser revista e atualizada continuamente.

Donabedian ${ }^{14}$, que tem se dedicado ao estudo da avaliação da qualidade há mais de vinte anos, apresenta um conceito unificado no qual expressa que o melhor cuidado é o que maximize o bem-estar do paciente, levando em conta o balanço dos ganhos e perdas esperados que acompanham o processo do cuidado em todas as etapas. Portanto, a assistência de qualidade implica em aumentar as probabilidades de êxito e reduzir aquelas consideradas como indesejáveis.

\section{Componentes da qualidade}

Segundo Donabedian ${ }^{14}$, a qualidade é uma idéia extremamente difícil de ser definida, considerando que a melhor definição de qualidade da assistência médica caracterizada através de seus componentes foi a proposta por Lee \& Jones, em 1933, expressa por meio de oito artigos de fé, válidos ainda no presente, a saber: fundamentação científica da prática médica; ênfase à prevenção; cooperação entre consumidores e prestadores de serviços; tratamento integral do indivíduo; relação estreita entre o médico e o paciente; serviços médicos integrais e coordenados; coordenação entre assistência médica e serviços sociais; acessibilidade da assistência a toda a população. 
Vuori ${ }^{21}$ comenta que a qualidade possui muitas facetas e que, atualmente, denota um grande espectro de características desejáveis de cuidados que incluem a efetividade, eficácia, eficiência, eqüidade, acessibilidade, adequação, aceitabilidade e qualidade técnico-científica.

A estas caracter:sticas, Gomes, citado por Arcucio e col ${ }^{1}$, acrescenta mais dois atributos: a continuidade dos cuidados e a comunicação eficaz entre o médico e o paciente.

Portanto, o significado de qualidade é abrangente, incluindo, freqüentemente, os aspectos de relacionamento interpessoal, de conforto proporcionado pelo cuidado e a eqüidade no acesso inicial aos serviços de saúde e na continuidade do atendimento. Já a finalidade da monitoração da qualidade é a de exercer uma vigilância, a fim de se detectar e corrigir, precocemente, os desvios encontrados, adotando-se, sempre, uma perspectiva pedagógica, e não punitiva.

\section{Padrões da Prática da Enfermagem}

A preocupação com a melhoria da qualidade da assistência de enfermagem tem levado as associações profissionais a estabelecerem padrões de cuidado que possam orientar a prática profissional e servir como instrumentos para monitorar e avaliar a assistência prestada.

Os padrões desenvolvidos para nortear a prática de enfermagem em Oncologia, propostos pela Sociedade de Enfermagem Oncológica dos Estados Unidos - ONS, em 1987, emergiram dos padrões para a prática de enfermagem estabelecidos, em 1973, pela Associação Norte-americana de Enfermeiras ${ }^{2}$.

Nos componentes constitutivos de cada padrão estão explicitados os critérios relacionados à estrutura, processo e resultados, de acordo com as abordagens de Donabedian ${ }^{13}$ para avaliação da qualidade em saúde.

Segundo Volker $^{20}$, o estabelecimento de padrões para a prática profissional tem por objetivo prover um guia para que as enfermeiras generalistas possam promover a qualidade da assistência por meio de identificação das bases técnicas que fundamentam o exercício profissional e pela aplicação do processo de enfermagem. No que se refere à prática da enfermagem oncológica, propicia uma base para a construção de instrumentos de avaliação, visando o controle da qualidade, estimulando a pesquisa para a fundamentação da prática, bem como a atuação inter e multidisciplinar. Para o cliente e familia, possibilita a participação nas atividades de promoção, manutenção e restabelecimento da saúde.

Tendo em vista a carência de estudos ou propostas de padrões para a prática de enfermagem em Oncologia em nosso meio, adotamos, para a análise do processo assistencial nesta investigação, as linhas gerais propostas por Ashley, Cross-Skinner ${ }^{2}$. 


\section{REFERENCIAL TEÓRICO}

Esta pesquisa tem por fundamento as abordagens de avaliação da qualidade em saúde propostas por Donabedian ${ }^{13}$, embasadas na Teoria de Sistemas.

Este autor propôs a triade Estrutura, Processo e Resultados como um referencial teórico para se processar essa avaliação .

O componente Estrutura corresponde aos recursos necessários ao processo assistencial, abrangendo a área física, pessoal (número, tipo e qualificação), recursos materiais e financeiros, sistema de informação e instrumentos normativos técnico-administrativos. Esta abordagem pressupõe que a presença de uma boa estrutura oferece as pré-condições para um bom desempenho nas áreas de processo e de resultado, e trabalha com informações bastante concretas e acessiveis.

O componente Processo abrange as atividades relacionadas à utilização dos recursos nos seus aspectos quanti-qualitativos e inclui o reconhecimento de problemas, métodos diagnósticos, o diagnóstico e os cuidados prestados. O pressuposto desta abordagem é $o$ de considerar correto quando os procedimentos adotados são coerentes, à luz do conhecimento científico vigente, quer na dimensão técnica, quer na relacional.

O componente Resultados corresponde às conseqüências das atividades do sistema de saúde ou do profissional em análise, em termos da melhoria do nível de saúde, da capacidade funcional restaurada ou alivio do sofrimento, como também a satisfação da clientela.

Segundo Paganini ${ }^{17}$, os enfoques possiveis para estudos avaliativos sobre a qualidade dos serviços de saúde podem ser centrados em um destes componentes, isoladamente, ou a partir da relação existente entre dois deles ou, ainda, entre os três, conforme demonstrado no esquema que se segue.

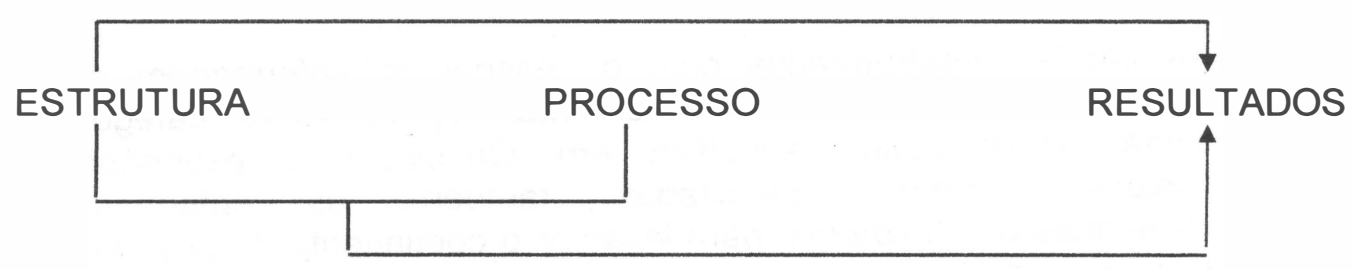

Nesta pesquisa, abrangeu-se a avaliação da estrutura existente em seis unidades de internação e na unidade de Quimioterapia Ambulatorial de Adultos, que atendem pacientes com câncer em um hospital universitário do Município de São Paulo, iniciando-se a avaliação do processo assistencial de enfermagem em quimioterapia prestado a tais pacientes, possibilitando relacionar a estrutura existente com alguns aspectos do processo de cuidar. 


\section{MATERIAL E MÉTODO}

\section{Local e Período de Estudo}

O trabalho de campo foi desenvolvido no hospital supracitado, classificado como um dos centros de referência estadual de nivel terciário para o tratamento de pacientes com câncer.

Esta instituição é categorizada como hospital geral, de porte extra (634 leitos), atende, principalmente, usuários do Sistema Único de Saúde, sendo campo de estágio para alunos da área de saúde, em níveis de graduação e pósgraduação lato sensu e stricto sensu.

Dentre as unidades de internação, foram selecionadas as de Ginecologia, Propedêutica I, Gastrocirurgia, Urologia, Pneumologia e Neurocirurgia, tendo, por critério básico, a distribuição proporcional da mortalidade por câncer no país em 1988, segundo as principais localizações anatômicas ${ }^{8}$ e, também, a unidade de Quimioterapia Ambulatorial de Adultos.

A coleta de dados foi realizada no período de janeiro a meados de junho de 1996.

\section{População}

A população deste estudo abrangeu:

- o pessoal de enfermagem que atua nas sete unidades selecionadas;

- a área física, instalações e os recursos materiais disponiveis nestas unidades para aplicação de drogas citostáticas;

- as normas técnicas relacionadas ao treinamento e à reciclagem desse pessoal para a prestação da assistência de enfermagem e à aplicação de quimioterapia antineoplásica;

- o sistema de informação sobre o câncer.

\section{Técnicas e Instrumentos de Coleta de Dados}

A coleta de dados foi realizada por meio de:

- entrevistas semi-estruturadas com o pessoal de enfermagem das unidades selecionadas, para obter informações sobre categoria funcional, qualificação específica em Oncologia e experiência profissional. Foram entrevistadas, também, as enfermeiras encarregadas das unidades, para levantar o contingente de pessoal e a existência de normas técnicas para aplicação da quimioterapia antineoplásica, e as enfermeiras responsáveis pelos setores de educação continuada e sistema de informação, visando obter dados sobre normas de treinamento e reciclagem dos funcionários de enfermagem e o sistema de informação em câncer;

- observação sistematizada de características da área física e das instalações dessas unidades, bem como dos processos de preparo e administração de drogas citostáticas; 
- observação sistematizada do processo assistencial de enfermagem a pacientes em quimioterapia antineoplásica, incluindo os aspectos técnicos e relacionais.

Os roteiros de entrevista e de observação foram elaborados com base em normas do Ministério da Saúde ${ }^{5,6,7}$ e Volker ${ }^{20}$, testados previamente, e modificados em aspectos que se mostraram necessários.

\section{APRESENTAÇÃO E DISCUSSÃO DOS RESULTADOS}

A Tabela que se segue objetiva mostrar a representatividade do câncer como causa de hospitalização nas unidades estudadas.

TABELA NÚMERO DE INTERNAÇÕES E PERCENTUAL DE PACIENTES COM DIAGNÓSTICO DE CÂNCER NO PERÍODO DE JANEIRO A MAIO, SEGUNDO AS UNIDADES ESTUDADAS DE UM HOSPITAL UNIVERSITÁRIO. SÃO PAULO, 1996.

\begin{tabular}{l|c|c|c}
\hline \multirow{2}{*}{ UNIDADES } & INTERNAÇÕES & \multicolumn{2}{|c}{ PACIENTES COM CANCER } \\
\cline { 3 - 4 } & No $^{\circ}$ & $\mathbf{N}^{\mathbf{2}}$ & $\%$ \\
\hline Ginecologia & 334 & 117 & 35,03 \\
Propedéutica I & 275 & 66 & 24,00 \\
Gastrocirurgia & 240 & 56 & 23,33 \\
Urologia & 166 & 24 & 14,46 \\
Pneumologia & 181 & 24 & 13,26 \\
Neurocirurgia & 240 & 27 & 11,25 \\
\hline \multicolumn{1}{c|}{ Total } & 1436 & 314 & $\mathbf{2 1 , 8 7}$ \\
\hline
\end{tabular}

Fonte: Caderno de registros das unıdades

No mesmo período, a unidade de Quimioterapia Ambulatorial de Adultos realizou 2154 atendimentos, dos quais $78,1 \%$ foram a portadores de câncer. A média diária de pacientes atendidos foi 33,6.

Nesta Tabela, pode-se observar que, no periodo considerado, os maiores percentuais de pacientes com câncer concentram-se em ordem decrescente, nas unidades de Ginecologia, Propedêutica e Gastrocirurgia. Esta representatividade é coerente com os dados de estimativa de incidência desta doença na Região Sudeste, que aponta como localizações mais freqüentes, no sexo feminino, a mama, o colo uterino e estômago e, no sexo masculino, o estômago e pulmão ${ }^{9}$. Segundo dados do Instituto Nacional de Câncer, os tumores malignos representam, atualmente, a segunda maior causa de morte por doença no país, perdendo apenas para doenças cardíacas .

A freqüência observada confirma, também, a configuração divulgada pelo Instituto Nacional de Câncer ${ }^{4}$ sobre a assistência ao paciente oncológico, que ocorre, predominantemente, em hospitais gerais, que oferecem serviços ambulatorial e de internação.

Esta realidade reforça a necessidade dos profissionais de saúde receberem, na sua formação básica e educação permanente, conteúdos relacionados à prevenção e detecção precoce do câncer, assim como ao tratamento e cuidados nos diferentes estágios desta doença. 
QUADRO ESTRUTURA: ÁREA FÍSICA E INSTALAÇÕES DAS UNIDADES DE INTERNAÇÃO ESTUDADAS DE UM HOSPITAL UNIVERSITÁRIO. SÃO PAULO, 1996.

\begin{tabular}{|c|c|c|c|c|c|c|c|c|c|}
\hline \multirow[b]{3}{*}{ Unidades } & \multirow{3}{*}{ Leitos } & \multirow[b]{3}{*}{$\begin{array}{c}\text { Área } \\
\mathrm{m}^{2}\end{array}$} & \multirow[b]{3}{*}{$\begin{array}{l}\text { Pias }^{3} \\
\text { No. }\end{array}$} & \multirow{2}{*}{\multicolumn{2}{|c|}{ Campainhas }} & \multicolumn{4}{|c|}{ Conjunto Sanitário dos Pacientes } \\
\hline & & & & & & \multicolumn{2}{|c|}{ sexo masculino } & \multicolumn{2}{|c|}{ sexo feminino } \\
\hline & & & & $\mathrm{N}^{0}$ & Funcionando & $\begin{array}{c}\text { Chuveiros } \\
\mathrm{N}^{0} \text {. }\end{array}$ & $\begin{array}{c}\text { Vasos Sanit. } \\
\text { NNo. }^{\circ}\end{array}$ & $\begin{array}{c}\text { Chuveiros } \\
\text { No }\end{array}$ & $\begin{array}{c}\text { Vasos Sanit. } \\
\text { NNo. }^{\circ}\end{array}$ \\
\hline $\begin{array}{l}\text { Pneumologia } \\
\text { Enfermarias: }\end{array}$ & & & & & & & & & \\
\hline $\begin{array}{c}\text { Entermarias: } \\
1\end{array}$ & 3 & 15,30 & 1 & 3 & 3 & 1 & 1 & & \\
\hline 2 & 3 & 16,00 & 1 & 3 & & & & 1 & 1 \\
\hline 3 & 3 & 16,10 & 1 & 3 & 3 & & & 1 & 1 \\
\hline 4 & 3 & 16,70 & 1 & 3 & 3 & 1 & 1 & & \\
\hline 5 & 3 & 16,10 & 1 & 3 & 3 & 1 & 1 & & \\
\hline UTI & 6 & 59,50 & 3 & & 0 & & & & \\
\hline${\text { Gastrocirurgia }{ }^{1}}$ & & & & & & 2 & 2 & 1 & 2 \\
\hline Enfermarias: & & & & & & & & & \\
\hline 1 a 6 & 18 & 17,00 & 1 & 3 & 3 & & & & \\
\hline 7 & 4 & 19,60 & 0 & 4 & 4 & & & & \\
\hline 8 & 3 & 17,60 & 1 & 3 & 3 & & & & \\
\hline 9 & 5 & 27,40 & 0 & 5 & 5 & & & & \\
\hline 10 & 3 & 18,00 & 0 & 5 & 3 & & & & \\
\hline$\frac{\text { Ginecologia }}{\text { Enfermarias: }}$ & & & & & & 0 & 0 & 2 & 2 \\
\hline $\begin{array}{c}\text { Enfermarias: } \\
1\end{array}$ & & & & & & & & & \\
\hline $\begin{array}{l}1 \\
2\end{array}$ & $\begin{array}{l}6 \\
4\end{array}$ & $\begin{array}{l}33,10 \\
21,25\end{array}$ & 1 & 4 & 4 & & & & \\
\hline 3 & 4 & 19,20 & 1 & 4 & 4 & & & & \\
\hline 4 & 4 & 18,78 & 1 & 4 & 4 & & & & \\
\hline 5 & 4 & 21,25 & 1 & 4 & 4 & & & & \\
\hline 6 & 3 & 16,50 & 1 & 3 & 3 & & & & \\
\hline Urologia & & & & & & & & & \\
\hline Enfermarias: & & & & & & 2 & 2 & 1 & 1 \\
\hline 1 e 2 & 6 & 3,13 & & 3 & 3 & & & & \\
\hline 3 e 4 & 6 & 2,89 & & 3 & 3 & & & & \\
\hline Propedêutica I & & & & & & & & & \\
\hline Enfermarias: & & & & & & 2 & 2 & 2 & 2 \\
\hline 1 a 9 & 27 & 16,80 & 1 & 3 & 3 & & & & \\
\hline 10 & 5 & 25,65 & & 5 & 5 & & & & \\
\hline 11 & 3 & 18,00 & & 3 & 3 & & & & \\
\hline 12 & 4 & 19,23 & & 4 & 3 & & & & \\
\hline Neurocirurgia & & & & & & & & & \\
\hline Enfermarias: & & & & & & 2 & 2 & 1 & 1 \\
\hline 1 a 6 & 18 & $16,80 \mathrm{~m}^{2}$ & 1 & 3 & 3 & & & & \\
\hline 7 a 8 & 4 & $16,80 \mathrm{~m}^{2}$ & 1 & 2 & 2 & & & & \\
\hline 9 & 2 & $15,30 \mathrm{~m}^{2}$ & 1 & 2 & 2 & & & & \\
\hline UTI & 5 & 42,00 & 1 & 5 & 5 & & & & \\
\hline Quimioterapia & & & & & & & & & \\
\hline 1 & $14^{2}$ & 59,64 & 1 & 0 & 0 & 0 & 0 & 0 & 0 \\
\hline
\end{tabular}

1 - 18 leitos para homens e 13 para mulheres

2 - Neste número foram incluidas 7 cadeiras ( tipo universitária), utilizadas para acomodar pacientes durante a aplicaçāo da quimioterapia

3 - Na Pneumologia fazem parte os conjuntos sanitários 
Os dados apresentados no Quadro anterior demonstram que as unidades de Propedêutica e Gastrocirurgia são as que possuem maior número de leitos (39 e 33, respectivamente). Nas unidades de internação, com exceção da Ginecologia, os leitos são distribuídos de forma homogênea, havendo predominância de três por enfermaria.

No que se refere à área em $\mathrm{m}^{2}$ dessas enfermarias, diante das atuais normas estabelecidas (BRASIL, 1994), que preconizam $6 \mathrm{~m}^{2}$ / leito em enfermarias de três a seis leitos, pode-se identificar que a área disponível nas unidades pesquisadas, é sempre menor do que o indicado. A exceção encontra-se na área da UTI de Pneumologia, bem próxima do recomendado, $10 \mathrm{~m}^{2}$ por leito. $\mathrm{Na}$ Quimioterapia, a área de $8,52 \mathrm{~m}^{2}$ / leito seria maior do que a recomendada se a área total não incluísse as sete cadeiras mencionadas, e locais destinados ao Posto de Enfermagem, Prescrição Médica, bem como ao preparo da medicação.

Em relação às instalações, a grande maioria das enfermarias está provida de pias e todas possuem campainhas funcionando normalmente, com acionamento em cada leito, o que facilita, respectivamente, a lavagem das mãos antes e após o cuidado prestado e o atendimento às solicitações dos pacientes ali internados. Com exceção da unidade de Pneumologia, de instalação mais recente no local em que se encontra, as outras não dispõem de conjuntos sanitários anexos às enfermarias, como é preconizado atualmente. Os existentes são destinados, com exclusividade, a pacientes de um ou outro sexo. Identificou-se carência de dois vasos sanitários na unidade de Ginecologia, uma vez que a norma preconiza um para seis leitos. A falta de sanitários na Quimioterapia representa desconforto para pacientes e respectivos acompanhantes, que necessitam usar os de uma unidade de internação localizada a certa distância no mesmo andar, e que já são insuficientes para atender seus próprios pacientes.

O Quadro a seguir demonstra, claramente, as inadequações da área física, entre as quais se ressalta a freqüência de área comum para as atividades desenvolvidas no Postó de Enfermagem e na Sala de Serviço, causando o aumento do fluxo de pessoas num mesmo local, prejudicando as condições de trabalho do pessoal de enfermagem e possibilitando o cruzamento de material limpo com o contaminado, o que pode causar aumento do índice de infecção hospitalar.

\section{QUADRO PRINCIPAIS CARACTERISTICAS DE ALGUNS ELEMENTOS DA ÁREA FISICA NAS UNIDADES ESTUDADAS DE UM HOSPITAL UNIVERSITÁRIO. SÃO PAULO, 1996.}

\begin{tabular}{|l|l|}
\hline ELEMENTOS & PRINCIPAIS CARACTERISTICAS \\
\hline $\begin{array}{l}\text { Posto de } \\
\text { Enfermagem }\end{array}$ & $\begin{array}{l}\text { Somente as unidades de Urologia e Propedêutica I possuem área } \\
\text { exclusiva }-12 \mathrm{~m}^{2} \text { e } 9,2 \mathrm{~m}^{2}, \text { respectivamente. } \\
\text { Nas unidades de Pneumologia, Ginecologia, Neurologia e Quimioterapia } \\
\text { funciona na mesma área da Sala de Serviço e na Gastrocirurgia está } \\
\text { situada no corredor em área de 5,92 } \mathrm{m}^{2} \text {, delimitada por balcőes. }\end{array}$ \\
\hline Sala de Serviço & $\begin{array}{l}\text { Apenas a unidade de Propedêutica I possui área física para este fim(7,92 } \\
\left.\mathrm{m}^{2}\right) \text { e na Gastrocirurgia está situada junto à copa. }\end{array}$ \\
\hline
\end{tabular}




\begin{tabular}{|l|l|}
\hline ELEMENTOS & PRINCIPAIS CARACTERISTICAS \\
\hline $\begin{array}{l}\text { Sala de Utilidades } \\
\text { ou Expurgo }\end{array}$ & $\begin{array}{l}\text { Somente a unidade de Pneumologia dispões de área física para este fim } \\
\left(15,2 \mathrm{~m}^{2}\right) \text {. Na Quimioterapia não existe este elemento e nas demais } \\
\text { unidades, a limpeza do material é realizada em área anexa aos conjuntos } \\
\text { sanitários dos pacientes. }\end{array}$ \\
\hline $\begin{array}{l}\text { Sala para Relatório e e } \\
\text { Prescrição Médica }\end{array}$ & $\begin{array}{l}\text { Nas seis unidades de internação são utilizadas bancadas situadas no } \\
\text { corredor interno. No Setor de Quimioterapia a escrivaninha do Posto de } \\
\text { Enfermagem é utilizada para este fim. }\end{array}$ \\
\hline $\begin{array}{l}\text { Sala de Exames e e } \\
\text { Curativos }\end{array}$ & $\begin{array}{l}\text { As unidades de Gastrocirurgia, Ginecologia e Urologia possuem salas } \\
\text { para este fim. }\end{array}$ \\
\hline Rouparia & $\begin{array}{l}\text { Não existe este elemento nas unidades estudadas. A roupa é } \\
\text { armazenada em armários situados no corredor, sendo estes, também } \\
\text { utilizados para a guarda de outros objetos. }\end{array}$ \\
\hline $\begin{array}{l}\text { Sanitários para } \\
\text { Funcionários }\end{array}$ & $\begin{array}{l}\text { Somente a unidade de Urologia possui conjunto de sanitário específico } \\
\text { para cada sexo. A Quimioterapia não dispõe deste elemento. }\end{array}$ \\
\hline
\end{tabular}

As deficiências observadas nos dois Quadros anteriores podem ser atribuídas à época de construção do hospital que está em funcionamento há mais de cinqüenta anos, às adaptações inadequadas e carência de recursos financeiros destinados a reformas das instalações.

É importante ressaltar que as inadequações do Setor de Quimioterapia foram solucionadas, mediante a concretização do projeto de instalação de uma nova área para este fim.

\section{Normas Técnicas Adotadas no Serviço de Enfermagem \\ Para treinamento e reciclagem}

Segundo informações obtidas com a enfermeira coordenadora do Serviço de Educação Continuada desse hospital, os funcionários recém admitidos recebem um treinamento inicial teórico-prático com duração de um mês, que aborda os procedimentos básicos de enfermagem e as Precauções-Padrão que devem ser adotadas na prestação de cuidados a todos os pacientes assistidos em serviços de saúde.

A programação da reciclagem é realizada anualmente, tendo por base necessidades identificadas pelas chefias, tanto para enfermeiras como para o pessoal auxiliar de enfermagem. São adotadas estratégias no sentido de atingir o maior número possível destes funcionários, nos três turnos de trabalho.

As informações obtidas permitiram detectar que, até o momento, não foi promovido, por esse Serviço, nenhum treinamento específico voltado para preparar o pessoal de enfermagem que vai atuar ou já trabalha na área de Oncologia, apesar dos tumores malignos representarem uma das principais causas de internação nesse hospital universitário. 


\section{Para a prestação da assistência de enfermagem}

O Serviço de Enfermagem possui, por escrito, normas que orientam a prestação de cuidados básicos de enfermagem, especificando, também, as Precauções-Padrão que devem ser obedecidas para evitar acidentes pessoais, tendo por base as normas propostas pelo Center for Diseases Control apud Carmagnani ${ }^{11}$.

A coordenadora do Serviço de Educação Continuada informou que o manual contendo estas normas encontra-se disponivel em todas as unidades de internação e ambulatoriais do hospital. No entanto, constatou-se que a unidade de Quimioterapia ainda não dispõe deste instrumento orientador.

\section{Para aplicação de quimioterapia antineoplásica}

As entrevistas realizadas com duas enfermeiras da Unidade de Quimioterapia e seis das unidades de internação permitiram conhecer que o tratamento quimioterápico é realizado tanto naquele setor, que atende pacientes adultos ambulatoriais, como nestas unidades.

A partir de novembro de 1996, foi instalada a Central de Quimioterapia, em local adaptado para esse fim, que, além de atender pacientes adultos em tratamento ambulatorial, se responsabiliza pelo preparo de drogas antineoplásicas, inclusive para pacientes internados. As novas instalações resultaram de reiterados esforços e reivindicações do grupo de enfermeiras e docentes de enfermagem que integram o Núcleo de Enfermagem em Oncologia(NEO), apoiado pelo Grupo Multidisciplinar de Oncologia, bem como pela Reitoria da Universidade e Diretoria de Enfermagem do Hospital. As instalações e equipamentos desta central atendem à normatização específica ${ }^{5}$.

As normas técnicas adotadas na unidade de Quimioterapia Ambulatorial de Adultos foram elaboradas pelo Comitê de Enfermagem da Fundação Oncocentro da Secretaria de Estado da Saúde de São Paulo, do qual fazem parte as duas enfermeiras entrevistadas. Essa normatização visa regulamentar a Portaria $\mathbf{N}^{\circ}$ 170/93, da Secretaria de Assistência à Saúde do Ministério da Saúde ${ }^{5}$, no que diz respeito às condições mínimas para a organização de uma central de quimioterapia.

No material escrito disponível nessa unidade, identificou-se que há descrição das normas técnicas referentes: ao preparo e administração de citostáticos; à segurança do pessoal de enfermagem que manipula estas drogas; às medidas preventivas para o descarte de material, proteção ambiental e pessoal; à previsão dos materiais e equipamentos a serem utilizados.

Entretanto, nas unidades de internação estudadas, verificou-se que estas não dispõem das normas orientadoras da segurança pessoal e de proteção do meio ambiente, assim como dos cuidados específicos para administração desse tipo de medicamentos.

No Gráfico seguinte pode-se perceber que, nas seis unidades de internação, há predomínio de auxiliares de enfermagem, figurando depois os enfermeiros. $\mathrm{Na}$ unidade de Quimioterapia, o número de enfermeiros é o mesmo que o dos 
auxiliares de enfermagem e não conta com atendentes no seu quadro de pessoal. A Diretoria de Enfermagem do hospital selecionado tem adotado a política de qualificar, profissionalmente, os atendentes, através da manutenção do Curso de Auxiliar de Enfermagem, obedecendo, assim, ao disposto na legislação que regulamenta o exercício profissional da enfermagem no país.

GRÁFICO Recursos Humanos de Enfermagem: - Distribuição do pessoal de enfermagem por categorias, segundo unidades estudadas de um hospital universitário - São Paulo, 1996

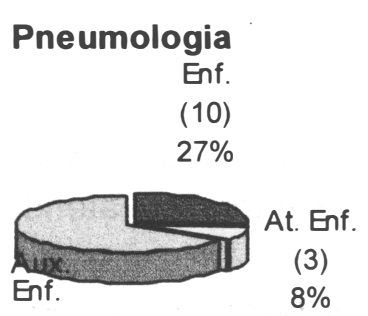

(24)

$65 \%$

Ginecologia

At. Enf.

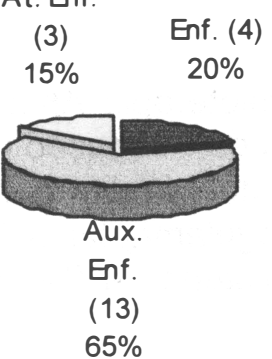

Neurocirurgia

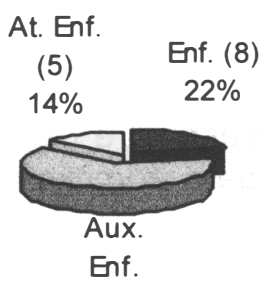

(24)

$64 \%$
Gastrocirurgia

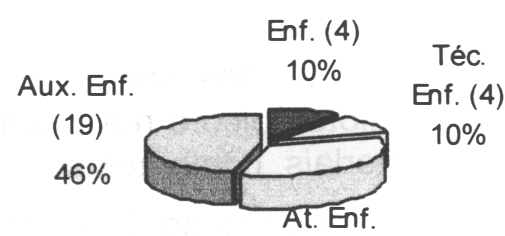

(14)

$34 \%$

\section{Propedêutica I}

Téc. Enf.

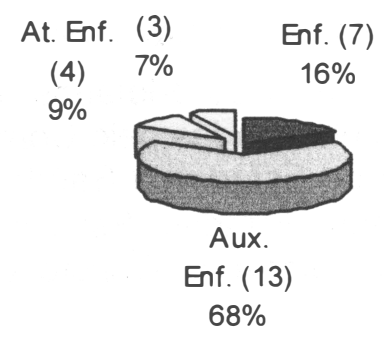

\section{Urologia}

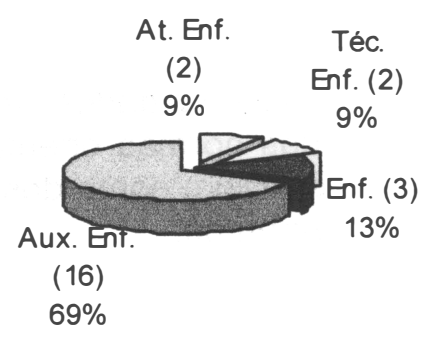




\section{Quimioterapia Ambulatorial de Adultos}

Téc. Enf.

$14 \%$

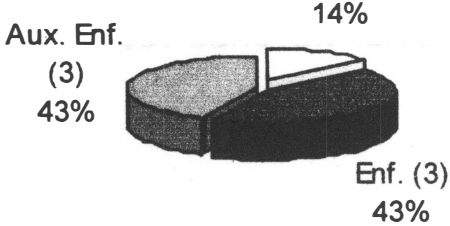

No que tange ao dimensionamento do pessoal de enfermagem, aplicou-se a fórmula adotada pelo setor de Educação Continuada da instituição selecionada e os resultados obtidos demonstram que há déficit de pesśoal em quatro das unidades estudadas e que o número de enfermeiros e de auxiliares de enfermagem é inferior ao recomendado em todas elas, em $14,4 \%$. No geral, o déficit de pessoal foi de $20,5 \%$.

Esta situação pode prejudicar a qualidade da assistência de enfermagem prestada aos pacientes internados, contribuindo também para a sobrecarga de atividades sob a responsabilidade desse pessoal, o que aponta para condições insatisfatórias de trabalho.

Quanto ao preparo em Oncologia, a grande maioria do pessoal de enfermagem não tem preparo específico nesta área, exceto o da Quimioterapia.

Dos 36 enfermeiros, 9 técnicos, 109 auxiliares e 31 atendentes de enfermagem das unidades de internação, apenas 11, 1, 8 e 1, respectivamente, possuem algum tipo de preparo específico em Oncologia. As modalidades de cursos freqüentados pelos enfermeiros foram: especialização em Estomaterapia, em Oncologia, cursos de curta duração sobre aspectos gerais desta especialidade ou sobre câncer ginecológico. O pessoal das demais categorias trabalhou anteriormente em hospitais especializados, onde recebeu treinamento em serviço sobre cuidados de enfermagem ao paciente com câncer. Estes dados mostram a necessidade do Setor de Educação Continuada inserir, nos programas de treinamento e de reciclagem, aspectos específicos da assistência ao paciente com câncer, tendo em vista que esta doença é um dos principais motivos de internação nesse hospital. Já, na Quimioterapia, a equipe de enfermagem possui preparo para esta área. As duas enfermeiras freqüentaram o Curso de Especialização em Enfermagem em Oncologia e uma participou de reciclagem nesta especialidade. Os demais membros desta equipe receberam treinamento teórico-prático, em serviço, sob a responsabilidade das enfermeiras da unidade.

No que se refere ao tempo de serviço, verificou-se predominância da faixa de 1 a 5 anos de trabalho em cinco unidades de internação, evidenciando que o pessoal de enfermagem possui experiência em assistir pacientes com câncer, 
necessitando, porém, de treinamento em serviço para integrar a teoria à prática. Seria necessário, também, incentivar enfermeiros a se especializarem nessa área, principalmente por se tratar de um hospital universitário, cuja finalidade é, além da assistência qualificada, o ensino e a pesquisa.

No entanto, do percentual de pessoal que trabalha há menos de um ano, não foi desprezivel, o que indica estar havendo rotatividade de funcionários nestas unidades.

\section{Recursos Materiais}

Segundo informações prestadas pelas duas enfermeiras do Setor de Quimioterapia, no período de março a meados de junho de 1996, ocorreu falta de cinco drogas citostáticas de última geração e uma adjuvante, todas de alto custo. Este fato causou interrupção e retardo do início de ciclos de tratamento, tanto dos pacientes externos como dos internados, com conseqüente prejuízo a clientes atendidos no hospital.

Esta situação é decorrente da grave crise financeira que afeta o Sistema Único de Saúde, responsável pelo atendimento à população de baixa renda no país, uma vez que o gasto per capita em saúde decresceu, no Brasil, de U\$95,9, em 1995, para U\$88,7, em 1997, enquanto em paises mais desenvolvidos este valor alcança U $\$ 1500^{19}$.

\section{Sistema de Informação}

O sistema de informação vigente no hospital em estudo está subdividido em duas partes, a saber: manual e informatizado. A ampliação da informatização depende da aquisição de novos computadores, capazes de armazenar maior número de informações. Nas unidades de internação foram implantados dois módulos: solicitação de medicamentos à farmácia (dose unitária) e resultados de exames laboratoriais.

O próximo módulo a ser implantado, em fase de elaboração, é a sistematização da assistência de enfermagem.

No que se refere ao registro hospitalar de câncer, este sistema foi implantado em novembro de 1996, visando integrar a instituição ao sistema de informações sobre câncer de âmbito nacional, já em funcionamento no país ${ }^{5}$.

\section{Processo assistencial em quimioterapia antineoplásica}

Os dados coletados permitiram identificar que, apenas no setor de Quimioterapia, o preparo de citostáticos é feito em capela de fluxo laminar, porém, sem exaustão externa e situada no mesmo ambiente onde ficam os pacientes, acompanhantes e pessoal de enfermagem. A revisão e troca de filtros desta capela é realizada com a periodicidade recomendada pelo fabricante e a limpeza obedece às normas técnicas 5 .

Nas unidades de internação, esse preparo ocorre em ambientes que não são exclusivos para este fim, como o posto de enfermagem, a sala de exames, ou 
até em uma das enfermarias, sobre um balcão ou mesa revestidos de fórmica. A limpeza desta superfície segue a rotina geral do serviço, sem os cuidados especiais preconizados para a administração de citostáticos.

Não foi observado o uso de oleado para proteção da área de preparo, em nenhuma das unidades estudadas.

Segundo informações obtidas, não é em todas as unidades que existe o conhecimento de que, na ocorrência de contaminação da superfície de preparo, esta deve ser limpa com álcool a $70 \%$; se as drogas citostáticas entram em contato com a pele, deve ser realizada a lavagem com água e sabão, e nos casos de contaminação ocular, além da irrigação, é necessário um exame oftalmológico. Quando o chão é contaminado, a limpeza é a de rotina, não sendo observados os cuidados especiais por se tratar de drogas citostáticas.

O preparo e administração das drogas são realizados por enfermeiras, técnicos e auxiliares de enfermagem e, nas unidades de internação, principalmente por esta última categoria. Ressalte-se, mais uma vez, o fato de que, com exceção do pessoal da unidade de Quimioterapia, a quase totalidade dos membros que executam tais atividades nas unidades de internação não recebeu nenhum tipo de programa de capacitação formal ou em serviço para atuar nesta área.

No que diz respeito às providências a serem tomadas na ocorrência de acidentes durante a manipulação de drogas citostáticas, segundo informações obtidas, em apenas duas unidades existe o conhecimento da existência e utilização de um impresso próprio, recentemente implantado para notificação de eventos adversos à Comissão de Controle de Qualidade, sendo também informado à chefia de enfermagem do setor. Em outra, a comunicação é feita apenas a esta chefia, nos casos de extravasamento de drogas durante a infusão da quimioterapia.

Em relação às normas de segurança profissional ${ }^{5}$, apenas o ambulatório de Quimioterapia possui normas escritas e atualizadas periodicamente, relacionadas aos riscos ocupacionais da manipulação de drogas antineoplásicas. Constatou-se, nas unidades estudadas, que nem todas as medidas preconizadas são adotadas, como, por exemplo, a paramentação completa, o descarte adequado do material perfuro-cortante, o afastamento de grávidas e nutrizes deste tipo de atividade, o exame semestral de saúde, incluindo avaliação clínica, exames hematológicos, provas de função hepática, renal e pulmonar.

Neste aspecto, são muito elucidativas as falas de duas auxiliares de enfermagem: Trabalho há 14 anos e sempre lidei com quimioterapia. Não tinha nenhuma noção do perigo e não faz muito tempo que comecei a ouvir falar dos riscos. Acho necessário um preparo especifico para isto, e mais controle dos funcionários que atuam nesta área. Eu mesma já tive plaquetopenia e leucopenia.

Aqui, uma auxiliar que estava grávida, afastou-se da quimioterapia porque ela mesma decidiu. Outra colega, pelo que me consta, atuava na quimioterapia durante a gravidez. 
Quanto às condições que determinam o encaminhamento de funcionários envolvidos com a quimioterapia para possível afastamento, apenas no setor de Quimioterapia foram citadas as três incluídas nas normas de segurança: $a^{5}$ lesões de pele, diarréia e gripe. Nas unidades de internação, houve referências isoladas a uma ou duas destas condições, sendo que em uma foi acrescentado o malestar geral. No entanto, foi relatado em uma das unidades que o funcionário encaminhado, não raro, é apenas medicado, sem ser concedido o afastamento do serviço, quando indicado.

Percebeu-se, durante as observações do processo assistencial em quimioterapia, que, embora a identificação dos problemas dos pacientes, o planejamento, a intervenção e a avaliação dos resultados da assistência prestada não sejam realizados de forma sistematizada e documentada, a interação do pessoal de enfermagem com os pacientes e familiares é permeada de atenção, respeito e consideração. Cabe lembrar que uma das metas da Diretoria de Enfermagem é a implantação da sistematização da assistência de enfermagem, de modo informatizado.

Constatou-se, também, a preocupação em adotar os padrões técnicos preconizados para a aplicação de drogas citostáticas, visando a segurança dos pacientes ${ }^{5,7}$.

Cabe ressaltar, ainda, que as enfermeiras reconhecem a interferência de problemas estruturais na assistência e têm procurado superá-los, mobilizando-se no sentido de conseguir melhorias indispensáveis para o processo de trabalho e promoção da qualidade da assistência de enfermagem prestada aos pacientes.

A recente instalação da Central de Quimioterapia em área física destinada a este fim, dispondo dos equipamentos necessários e de pessoal de enfermagem qualificado para o desempenho das atividades de preparo e administração de drogas citostáticas, certamente contribuirá para a melhoria da qualidade do atendimento prestado ao paciente com câncer, bem como aos familiares.

No entanto, apesar de uma gerência eficaz, alguns problemas de âmbito macroestrutural, tais como o baixo financiamento do setor público de saúde e a política de recursos humanos deste setor, concretizados nos baixos salários e na deterioração das condições de trabalho, geram a insatisfação e a rotatividade do pessoal de enfermagem, com repercussões na qualidade da assistência prestada ao paciente.

\section{CONCLUSÕES}

A avaliação da estrutura existente nas unidades estudadas demonstrou deficiências na área física e instalações, falta de medicamentos citostáticos em determinado período, o que aponta para a não manutenção da qualidade desejada no atendimento ao paciente com câncer no hospital universitário, campo do estudo. Quanto ao dimensionamento do pessoal de enfermagem, 
constatou-se carência de profissionais, principalmente de enfermeiros. Apesar da existência de um processo de educação continuada, não há programas específicos para o treinamento e atualização na área de Oncologia. A exceção se apresenta, apenas, no Ambulatório de Quimioterapia de Adultos, onde as enfermeiras são especialistas e se responsabilizam pelo preparo dos demais membros da equipe de enfermagem dessa unidade.

No que se refere à sistematização da assistência de enfermagem, observouse que não há um método implantado, mas existe um esforço neste sentido por parte da diretoria de enfermagem do hospital.

Grande parte dos problemas identificados decorrem da política de saúde adotada no país, que tem se refletido nas diversas carências presentes nas instituições públicas de saúde.

\section{REFERÊNCIAS BIBLIOGRÁFICAS}

1. ARCUCIO, F.A. et al. Avaliação da qualidade de serviços de saúde. Saúde em Debate, v.33, p.50-53, 1991.

2. ASHLEY, B. W.; CROSS-SKINNER, S. Oncology Nursing Care Delivery Issues in the Ambulatory Setting. In: HUBBARD, S.M.; GREENE, P.E.; KNOBF, M. T. Current Issues in Cancer Nursing Practice Updates, v.1, p.1-10, 1992.

3. AZEVEDO, A.C. Avaliação do desempenho de serviços de saúde. Rev. Saúde Públ., São Paulo, v.25, n.1, p.64-71, 1991.

4. BRASIL. Ministério da Saúde. Instituto Nacional de Câncer. Câncer no Brasil: a magnitude do problema e medidas de controle, Rio de Janeiro: INCA/Pro-Onco, 1990.

5. BRASIL. Portaria $N^{\circ}$. 170, de 17 de dezembro de 1993. Estabelece normas para credenciamento de hospitais que realizam procedimentos de alta complexidade em Câncer. Diário Oficial da República Federativa do Brasil, Brasília, v.241, p.19.753-58, 20 dez. 1993. Seção 1.

6. BRASIL. Ministério da Saúde. Secretaria de Assistência à Saúde. Departamento de Normas Técnicas. Normas para projetos físicos de estabelecimentos assistenciais de saúde. Brasília: DNT, 1994.

7. BRASIL. Ministério da Saúde. INCa/PRO-ONCO - Ações de enfermagem para o controle do câncer. Rio de Janeiro: INCa/PRO-ONCO, 1995a.

8. BRASIL. Ministério da Saúde. Instituto Nacional de Câncer. O problema do câncer no Brasil. 3. ed., Rio de Janeiro: INCAVPro-Onco, 1995b. 
9. BRASIL. Ministério da Saúde. Secretaria Nacional de Assistência à Saúde. Instituto nacional de Câncer. Coordenação de Programas de Controle de Câncer - PRO-ONCO. Estimativa da Incidência e Mortalidade por Câncer no Brasil 1996. Rio de Janeiro: Pro-Onco, 1996. 19p.

10. CAMPOS, F.E. Resolutividade: uma aproximação à avaliação qualitativa dos serviços de saúde. Belo Horizonte: Imprensa Universitária, 1988.

11. CARMAGNANI, M.I.S. (Coord.) Manual de procedimentos básicos de enfermagem. Rio de Janeiro: Interlivros, 1995. 312p.

12. CORDERA, A. BOBENRIETH, H. Administración de los servicios de salud. México: Interamericana, 1983. p.529-67

13. DONABEDIAN, A. Explorations in quality assessment and monitoring. Michigan: Health Administration Press, 1980.

14. DONABEDIAN, A. Evaluación de la calidad de la atención médica. In: WHITE, K.L.(coord.) Investigaciones sobre servicios de salud: una antologia. Washington, D.C.: OPS, 1992. p. 128 (Public.Cient. 534).

15. EDITORIAL. Nuevos horizontes de la calidad de la atención a la salud. Salud públ. México, v. 35, n. 3, p.235-37, mayo/jun., 1993.

16. NOVAES, H.M. Programas de garantia de calidad através de la acreditación de hospitales en Latino América y Caribe. Salud Públ. México, v.35, n.2, p.148-258, 1993.

17. PAGANINI, J.M.. Calidad y eficiencia de la atención hospitalaria. Washington, D.C.: OPS, 1993. (HSS/SILOS: 30)

18. REIS, E.F.B. et al. Avaliação da qualidade dos serviços de saúde - notas bibliográficas. Cad. Saúde Pública, v.6, n.1, p.50-61, 1990.

19. SÚMULA. Radis, Rio de Janeiro, n. 58, abr., 1996

20. VOLKER, D.L. Padrões da prática da enfermagem oncológica. In: CLARK, J.C.; McGEE, R.F. Enfermagem Oncológica - um currículo básico. Porto Alegre: Artes Médicas, 1997.

21. VUORI, H. A qualidade da saúde. Saúde em Debate, n.3, p.1725, 1991. (Caderno de Ciência e Tecnologia, 1) 